\title{
Thermally Actuating Multifunctional Molecular Crystals
}

\section{Durga Prasad Karothu', Rodrigo Ferreira ${ }^{1}$, Luca Catalano ${ }^{1}$, Ghada Dushaq ${ }^{2}$, Mahmoud Rasras ${ }^{2}$, Panče Naumov ${ }^{1}$}

${ }^{1}$ Department of Chemistry, New York University Abu Dhabi, Saadiyat Island, Abu Dhabi, UAE, ${ }^{2}$ Department of Electrical and Computer Engineering, New York University Abu Dhabi, Saadiyat Island, Abu Dhabi, UAE

dpk3@nyu.edu

There has been a growing interest in mechanically responsive molecular crystals that show reversible and unusually large positive and negative thermal expansion triggered by external stimuli, a property which could be applied to the design of actuators for soft robotics, artificial muscles, and microfluidic and electrical devices [1]. However, controlling molecular motion to execute sufficiently larger and practically useful thermal expansion in crystals remains a formidable challenge, and strong deformation of such crystals usually results in their destruction [2]. Here we report a single crystal of simple organic compound which exhibits giant thermal expansion due to collective reorientation of molecules in the crystal lattice which is reversible after more than fifty heating and cooling cycles. Such atypical molecular motion, revealed by single crystal X-ray diffraction and microscopy analyses, drives an exceptionally large expansion of the crystal. The applicability of the material as an actuator with electrical properties is demonstrated by dielectric, capacitance, conductance and current measurements. The large shape change of the crystal, combined with remarkable durability and electrical properties, suggest that this material is a strong candidate for microscopic multifunctional thermal actuating devices.

[1] Naumov, P., Chizhik, S, Panda, M. K, Nath, N. K., Boldyreva, E. (2015). Chem. Rev. 115, 12440.

[2] Colin-Molina, A., Karothu, D. P., Jellen, M. J., Toscano, R. A., Garcia-Garibay, M. A., Naumov, P., Rodríguez-Molina, B. (2019). Matter. 1, 627.

Keywords: Smart materials, Soft crystals, thermal actuators, Crystal adaptronics. 